\title{
Statins and risk of breast cancer recurrence
}

Minas Sakellakis'

Karolina Akinosoglou'

Anastasia Kostaki ${ }^{2}$

Despina Spyropoulou'

Angelos Koutras'

'Department of Medicine, Division of Oncology, University Hospital, Patras Medical School, Patras, ${ }^{2}$ Department of Statistics, Athens University of Economics and Business, Athens, Greece
Correspondence: Minas Sakellakis Department of Medicine, Division of Oncology, University Hospital of Patras, I Leoforos Ippokratous, Rion, Patras 26504, Greece

$\mathrm{Tel}+302610999535$

Fax +30 26I 0994645

Email doctorsakellakis@gmail.com
This article was published in the following Dove Press journal:

Breast Cancer - Targets and Therapy

4 November 2016

Number of times this article has been viewed

Background: The primary end point of our study was to test whether the concurrent use of a statin is related to a lower risk of recurrence and increased relapse-free survival in patients with early breast cancer.

Materials and methods: We reviewed 610 female patients with stage I, II, or III breast cancer who had been surgically treated and who had subsequently received at least adjuvant chemotherapy in order to prevent recurrence.

Results: Among the 610 patients with breast cancer, $83(13.6 \%)$ were receiving a statin on a chronic basis for other medical purposes. Overall, statin users displayed longer mean relapsefree survival ( 16.6 vs 10.2 years, $P=0.028$ ). After data had been adjusted for patient and disease characteristics, statin users maintained a lower risk of recurrence. This favorable outcome in statin users was particularly evident when we included only younger patients in the analysis ( 20 vs 10 years, $P=0.006$ ).

Conclusion: Statins may be linked to a favorable outcome in early breast cancer patients, especially in younger age-groups.

Keywords: statins, breast, cancer, adjuvant, recurrence

\section{Introduction}

Statins are widely used in primary and secondary prevention of cardiovascular diseases, because of their significant capacity to lower serum cholesterol levels. ${ }^{1}$ Statins reduce cholesterol biosynthesis in the liver by interfering with the mevalonate pathway and inhibiting HMGCR. In addition to cholesterol, this pathway produces isoprenoids, which are necessary for regulation of cell growth and oncogene expression, ${ }^{2-9}$ while cholesterol is a critical component of intracellular lipid rafts, which are pivotal for intracellular signaling. ${ }^{9}$ Apart from their role as lipid-lowering agents, they have been found to have immunomodulatory and antineoplastic effects. ${ }^{1,10-12}$ Several preclinical studies have suggested a direct effect of these agents in certain cellular functions, including cell-cycle arrest and apoptosis, ${ }^{13,14}$ cancer invasion and angiogenesis, ${ }^{15,16}$ tumor differentiation or sensitization to chemotherapy, and cholesterol formation. ${ }^{17-19}$ Several studies have found a reduced risk of developing various types of cancer, including breast cancer, in patients using statins. ${ }^{1,20-23}$ Benefit has recently been expanded to patients already diagnosed with cancer receiving statins compared to nonusers, showing reduced cancer-related mortality. ${ }^{24,25}$ In patients with breast cancer, large epidemiologic studies have recently investigated the effect of statin use following cancer diagnosis on disease progression. ${ }^{26-30}$ Though promising, results remain inconclusive, and further studies are needed in order 
to answer the question definitively. ${ }^{31,32}$ The aim of our study was retrospectively to assess a possible correlation between chronic statin use in the adjuvant setting and disease-free survival (DFS) in patients with stage I-III breast cancer.

\section{Materials and methods}

We performed a retrospective search of the medical archive at our oncology department. We reviewed all cases with a history of early stage breast cancer that were treated in our department from 1983 to 2013 . We defined as "early stage" patients with stage I, II, or III disease that had been surgically treated and who had received at least adjuvant chemotherapy in order to prevent recurrence. We excluded patients with locally advanced disease at the time of initial diagnosis who had undergone neoadjuvant chemotherapy and patients with residual disease after surgery. We also excluded male patients and patients who did not receive chemotherapy. After an initial screening, 934 patients were eligible for further review. After a thorough evaluation of each medical record, we extracted the following parameters for each patient: age, tumor stage according to the American Joint Committee on Cancer Staging Manual (sixth edition), hormonal receptor status (if either estrogen or progesterone receptors were $>1 \%$ expressed), HER2 status, and whether they were receiving a statin on a chronic basis or not. Information regarding statin use was obtained during the initial visit. However, we included only patients who were on chronic statin therapy. Whenever relevant information was not present, those cases were excluded. Although these patients usually intend to receive lifelong statin therapy, the fact that we did not monitor statin administration over time constitutes a limitation of our study.

The primary end point of our small study was to test whether the concurrent use of a statin was associated with increased cancer-specific DFS. Statins in our patients were used mostly for dyslipidemia on a chronic basis. In all cases, the goal of statin administration was to prevent cardiovascular events. For each patient, we documented the time period (counted in years) in which they had a disease relapse, starting from the day the patient initiated chemotherapy. Whenever the patient did not relapse, we documented the overall time until the last documented surveillance visit (censored data). Whenever the patients had died due to reasons other than cancer, they were recorded as not relapsed (censored data). Survival analysis was performed, and we compared the two groups (statin users versus non users) in terms of DFS. We fitted Cox proportional hazard ratio (HR) models before and after adjusting for confounding factors, and we extracted our results using HRs and 95\% confidence intervals (CIs). When multiparametric analysis was performed, all possible models containing all possible combinations of variables were compared, based on the $-2 \log$-likelihood value and the number of variables included. $P$-values $<0.05$ were used to define statistical significance. In all comparisons, we used two-sided tests. Kaplan-Meier curves were plotted, and we also used log-rank tests for comparisons. In the present study, we did not address the effect of statins on overall survival. All data were analyzed using IBM SPSS software Version 20.0 (IBM Corporation, Armonk, NY, USA). Ethical approval was provided by the institutional review board of the University Hospital of Patras, Greece. Written informed consent waived by the review board because of the retrospective chart review nature of the study that involved subjects over a 30-year period, many of whom passed away or were lost to follow-up.

\section{Results}

After the final screening, a total of 610 of 934 patients were eligible for further evaluation. Among 324 patients who were excluded, the majority (approximately two-thirds of excluded patients) of them had no clear information in the medical records regarding statin use or the patients were using statins for a limited time. The rest were excluded due to missing information regarding the outcome. A small portion of cases were excluded because of insufficient data regarding whether they successfully completed the recommended adjuvant therapy.

The median age of the patients was 56 years. For practical purposes, we divided our patients into two age-groups, depending on whether they were younger or older than 56 years. Among 610 patients with breast cancer, 83 (13.6\%) were receiving a statin. Most frequently used regimens included atorvastatin (45 patients), simvastatin (19), pravastatin (eleven), rosuvastatin (six), and fluvastatin (two).

Baseline patient characteristics are shown in Table 1. The mean age of patients who received statins was 64 years, while the mean age for nonusers was 55 years $(P<0.001)$. There were no significant differences with respect to tumor

Table I Baseline patient characteristics among statin users and non-statin users

\begin{tabular}{llll}
\hline Characteristics & No statins & Statins & Significance \\
\hline $\begin{array}{l}\text { Mean age (years) } \\
\text { Clinical stage }\end{array}$ & 55 & 64 & $<0.00$ I \\
I & $62(15 \%)$ & $15(20 \%)$ & 0.506 \\
II & $24 I(60 \%)$ & $33(45 \%)$ & \\
III & $100(25 \%)$ & $26(35 \%)$ & \\
Hormonal status & & & \\
Positive & $206(85.5 \%)$ & $35(84.5 \%)$ & 0.545 \\
Negative & $35(14.5 \%)$ & $6(15.5 \%)$ & \\
HER2 & & & \\
Positive & $72(29 \%)$ & $8(23 \%)$ & 0.369 \\
Negative & $177(71 \%)$ & $27(77 \%)$ & \\
\hline
\end{tabular}


stage ( $P=0.506)$, apart from the fact that more patients in the statin group were at clinical stage III $(P=0.033)$. Additionally, there were no differences among statin users and nonusers with respect to hormonal receptor status $(P=0.545)$ or HER-2 status $(P=0.5441)$.

Median follow-up for statin users was 24 (3-336) months, while that for nonusers was 48 (2-360) months. Statin users had a mean overall DFS of 16.6 years and median DFS of 20 years. On the other hand, those not receiving a statin had 10.2 and 9 years, respectively (log-rank test, $P=0.028$ ) (Table 2; Figures 1 and 2). This favorable outcome observed in statin users was particularly evident when we included only younger patients in the analysis ( 20 vs 10 years, $P=0006$ ) (Table 3). On the other hand, there was no difference when we included only older patients in the analysis ( 9 vs 9.2 years, $P=0.456)$. A marginal association was also noted when we included only the hormone receptor-positive subgroup (10 vs 18 years, $P=0.024$ ) (Table 4). No comparisons in other

Table 2 Cox proportional HR-model results of statin effect, after adjusting for various parameters

\begin{tabular}{|c|c|c|c|c|c|c|}
\hline $\begin{array}{l}\text { Statin effect } \\
\text { adjusted for }\end{array}$ & Events & Censored & HR & $95 \% \mathrm{Cl}$ & $P$-value & -2Log likelihood \\
\hline Age-Stage & 154 & 301 & 0.7 & $0.4-1.2$ & 0.202 & $\mathrm{I}, 558$ \\
\hline Age-Stage-HR & 53 & 136 & 0.67 & $0.28-1.6$ & 0.382 & 432 \\
\hline Age-Stage-HR-HER & 40 & 128 & 0.39 & $0.11-1.4$ & 0.134 & 318 \\
\hline Stage-HR-HER & 40 & 128 & 0.4 & $0.1 \mathrm{I}-\mathrm{I} .4$ & $0 .|4|$ & 318 \\
\hline Stage-HR & 53 & 136 & 0.68 & $0.28-1.6$ & 0.393 & 432 \\
\hline Stage-HER & 40 & 129 & 0.43 & $0.12-1.4$ & 0.172 & 318 \\
\hline Stage & 154 & 303 & 0.34 & $0.30-1.2$ & 0.235 & $\mathrm{I}, 560$ \\
\hline Age & 243 & 338 & 0.55 & $0.34-0.9$ & 0.016 & 2,553 \\
\hline HR & 105 & 157 & 0.51 & $0.25-1.1$ & 0.74 & 913 \\
\hline HER & 68 & 188 & 0.53 & $0.22-1.4$ & 0.155 & 548 \\
\hline
\end{tabular}

Abbreviations: $\mathrm{HR}$, hazard ratio; $\mathrm{Cl}$, confidence interval.

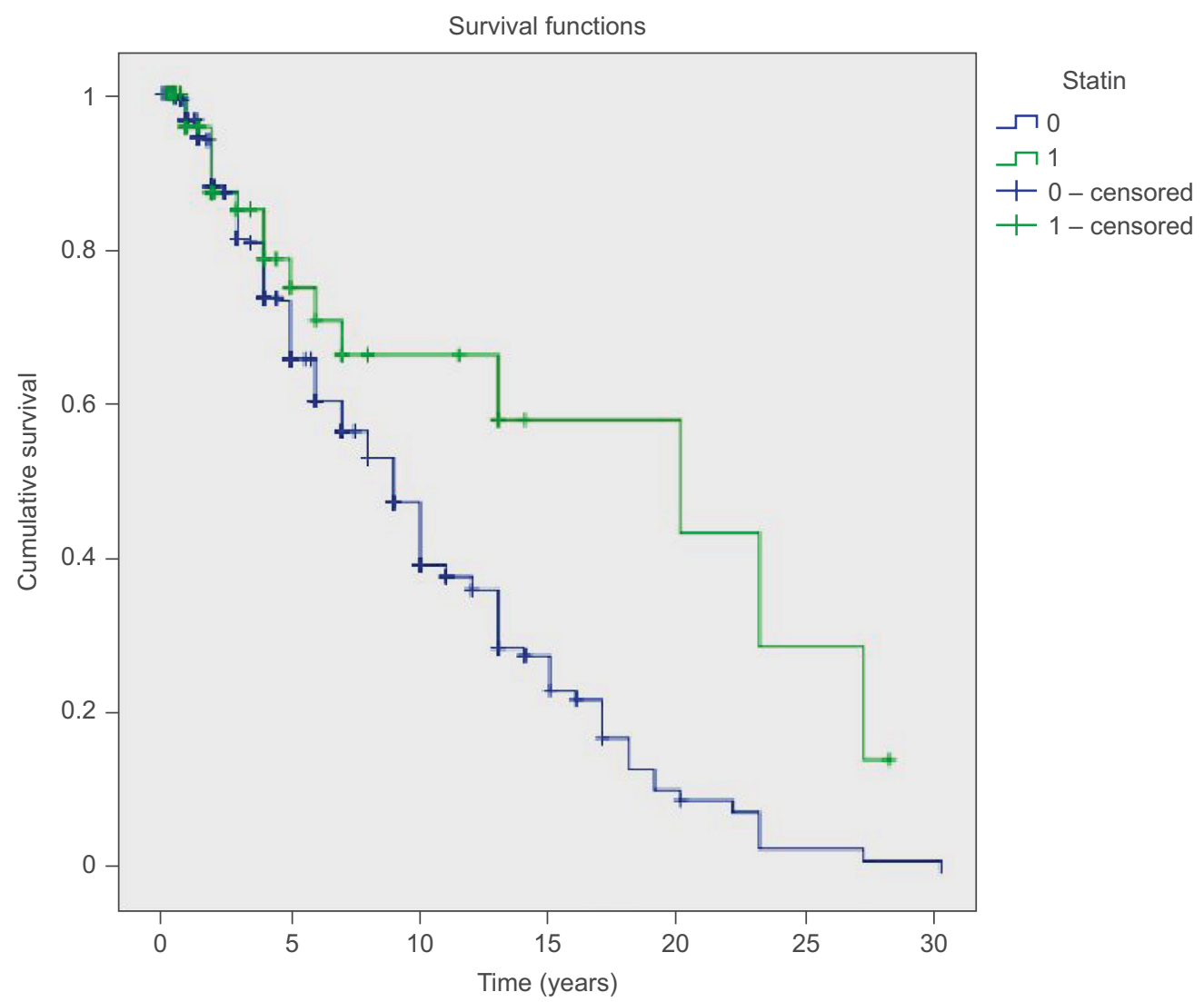

Figure I Kaplan-Meier curves for patients with breast cancer.

Notes: The green line represents the cumulative disease-free survival of 83 statin users, while the blue line represents cumulative disease-free survival of 527 non-statin users. 


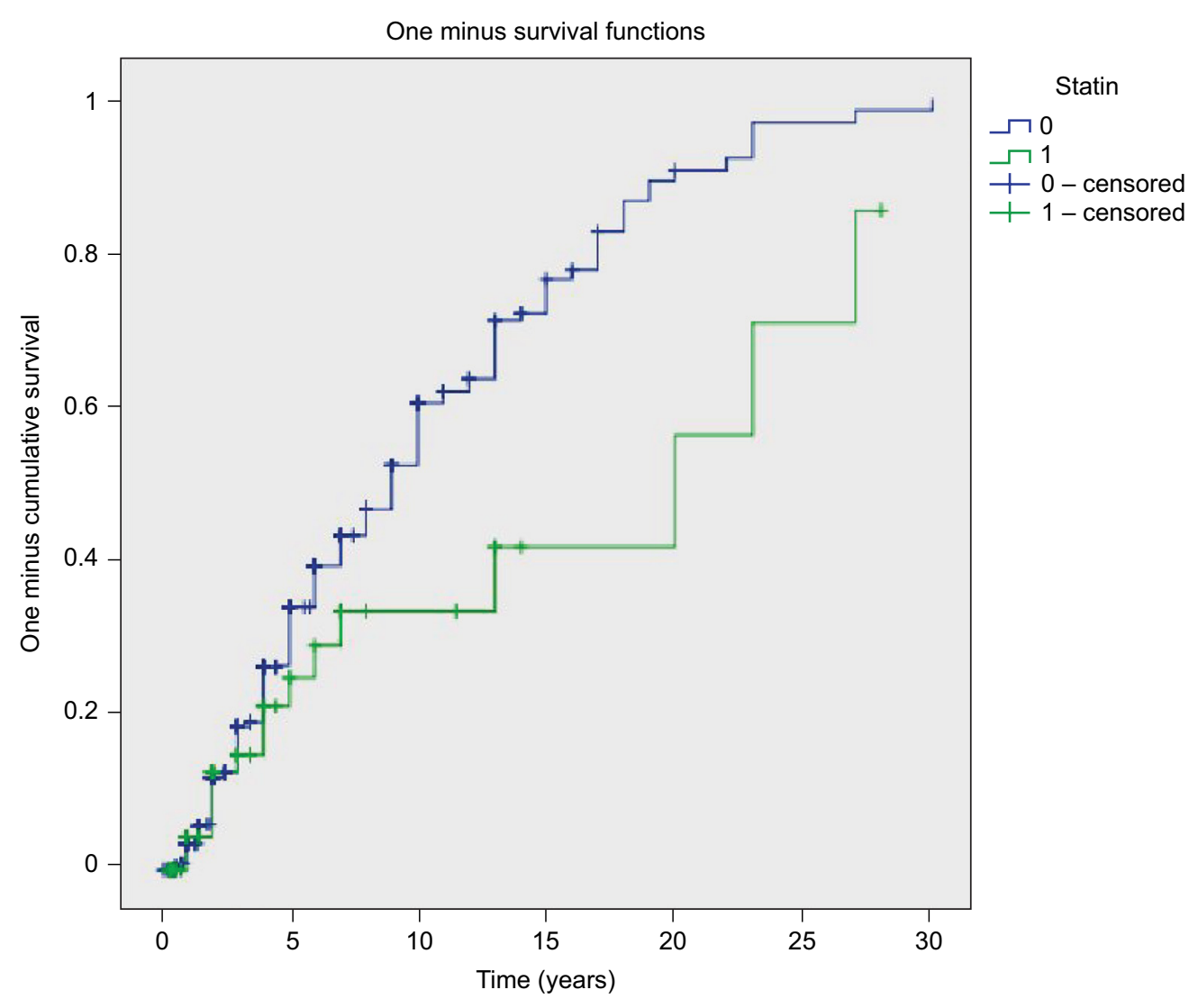

Figure 2 Kaplan-Meier curves displaying proportion of patients at risk over time.

Notes: The green line represents the 83 statin users, while the blue line represents the 527 non-statin users.

Table 3 Cox proportional HR-model results of statin effect when only patients under the median age were included in the analysis

\begin{tabular}{llllll}
\hline $\begin{array}{l}\text { Statin effect } \\
\text { adjusted for }\end{array}$ & Events & Censored & HR & $\mathbf{9 5 \%} \mathbf{C l}$ & P-value \\
\hline Stage & 76 & 153 & 0.4 & $0.1-1.4$ & 0.102 \\
HR & 65 & 76 & 0.37 & $0.1-1.1$ & 0.056 \\
HER & 42 & 89 & 0.35 & $0.1-1.2$ & 0.1 \\
\hline
\end{tabular}

Abbreviations: $\mathrm{HR}$, hazard ratio; $\mathrm{Cl}$, confidence interval.

Table 4 Cox proportional HR-model results of statin effect when only patients with hormone receptor-positive tumors were included in the analysis

\begin{tabular}{llllll}
\hline $\begin{array}{l}\text { Statin effect } \\
\text { adjusted for }\end{array}$ & Events & Censored & HR & $\mathbf{9 5 \%} \mathbf{C l}$ & P-value \\
\hline Age & 90 & 132 & 0.4 & $0.18-1$ & 0.047 \\
Stage & 44 & 111 & 0.5 & $0.17-1.6$ & 0.24 \\
HER & 45 & 119 & 0.4 & $0.13-1.1$ & 0.082 \\
\hline
\end{tabular}

Abbreviations: $\mathrm{HR}$, hazard ratio; $\mathrm{Cl}$, confidence interval.

subgroups revealed any significant beneficial correlations; however, the number of patients in each subgroup was too small to draw definite conclusions, and the results should be interpreted with extreme caution.

In this small study, we found that the concurrent administration of statins in the adjuvant setting might be linked to a favorable outcome, as statin users had a lower risk of relapse (HR 0.58; 95\% CI 0.36-0.94; $P=0028$ in univariate analysis) (Table 2). Multivariate analysis showed the optimal model best fitting the data was the one that included only patient age (HR 1.01; 95\% CI 1-1.023; $P=0.044$ ) and statin administration (HR 0.55; 95\% CI 0.34-0.89; $P=0.016$ ). Multivariate analysis results are provided in Table 2. Despite the effect of age, statin use independently predicted a better outcome. In conclusion, although far from proving causality, our study indicates that statins might possibly be related to a better outcome in younger patients and those with tumors expressing hormone receptors. However, as stated, the results should be interpreted cautiously, particularly the subgroup analyses, due to small sample size.

\section{Discussion}

The present study investigated the relationship between statins and breast cancer recurrence in terms of DFS, involving 610 patients extracted from our medical records in a period of 30 years. Statin use was found to be associated with increased overall DFS, in line with previous studies exploring the impact of statin use in breast cancer patients. ${ }^{28,33-36}$ Pre- or postdiagnosis statin studies have already shown a beneficial effect on survival ${ }^{34,36}$ but not all of them..$^{28,33,35}$ Even in the latter case, where the studies showed no effect on survival, 
patients receiving statins, for more than 6 months $s^{28}$ or receiving weakly lipophilic to hydrophilic statins ${ }^{33,34}$ exhibited significantly improved DFS. Similarly to our results, lower rates of recurrence have been reported in the past. ${ }^{26,28-30} \mathrm{In}$ a retrospective cohort of 703 females with stage II or III breast cancer $^{28}$ and in a prospective cohort of 1,945 females with stage I-III breast cancer, ${ }^{29}$ lower risk of cancer recurrence was observed in statin-treated patients. Interestingly, in a study of 18,769 patients, benefit was observed only among females with ER-positive breast cancer ${ }^{26}$ in agreement with our results. Similarly, large population-based studies of patients with stage I-III breast cancer receiving lipidlowering regimens including statins confirmed a decreased risk of recurrence, ${ }^{30}$ as well as lower overall cancer and breast cancer-specific mortality among statin users. ${ }^{25}$

Studies suggest that the "statin effect" was stronger in tumors that were positive for hormone receptors. This suggests a possible interaction between hormonal pathways and statin activity that tends to modulate the effects of female reproductive hormones to cancer cells, probably by intervening in the regulation of the cell cycle and induction of cell death. ${ }^{26,37} \mathrm{It}$ is known that both estrogen receptors and statins play a role in tumor neoangiogenesis, the latter by reducing the production of VEGF ${ }^{38,39}$ similarly to tamoxifen. ${ }^{38}$ Furthermore, estrogen receptors are known to enhance the activation of the Fas-ligand promoter, ${ }^{40}$ while on the other hand statins reduce Fas-ligand expression on tumor cells, thus altering its resistance to cellular immunity. ${ }^{41}$ Our finding is consistent with previous research ${ }^{26}$ supporting this association; however, whether statins possibly exert this effect solely in metabolic pathways by preventing ER stimulation ${ }^{42}$ or de novo breast cancer phenotypic conversion from ER-negative to ER-positive similarly to other agents ${ }^{43}$ remains to be elucidated. ${ }^{44,45}$ Of note, of unknown but intriguing significance remains the fact that a number of studies have associated HMGCR expression (statin pharmaceutical target) with ER expression., ${ }^{7,46}$

Our results also suggest a possible favorable link in females of younger age. Preclinical and epidemiological studies have shown that cancer in this group is a separate entity with unique characteristics ${ }^{47}$ that carries a worse prognosis. ${ }^{48,49}$ Experiments have shown different patterns of gene expression in pathways that constitute hallmarks of cancer progression, such as apoptosis, hypoxia-induced response, inflammatory response, and such pathways as Myc, E2F, Ras, and mTOR. ${ }^{47}$ All this can constitute possible targets of statin activity; however, in previous studies no further analysis upon age distribution was performed to allow for further assumptions.

Except for the several primary cancer-preventive mechanisms already described, statin effects in breast cancer could be attributed to a number of existing hypotheses. Perioperative trials using neoadjuvant statin therapy have shown reduced tumor-proliferation rate ${ }^{50}$ in potential relation to HMGCR expression, ${ }^{51}$ which in turn is associated with better prognosis. ${ }^{7}$ Moreover, in vitro experiments have suggested that statins stimulate production of a variant of the p53 transcription factor and decreased the potential of bone metastasis, ${ }^{16}$ although this has not been confirmed in the clinical setting. ${ }^{26}$

\section{Limitations}

Limitations of our study include the low prevalence of statin use among patients and lack of data on medication compliance among study participants. In addition, there is no information regarding tumor size, grade, lymph-node status, etc, but due to the small number of cases, we preferred not to include all those multiple parameters, which would dramatically decrease the power of multiparametric statistical analysis, making it virtually useless with so many variables in a small sample. Therefore, we chose to include them all under the tumor-stage umbrella. Moreover, information is limited upon statin dose and exact duration of administration (although reported at the initial visit that it was taken on a chronic basis and intended to be continued lifelong), recently reported to affect outcome. ${ }^{28,35}$ No adjustment was made with regard to type of statin administered. As previously shown, the lipophilic statins have an increased ability to penetrate cell membranes, and hence are responsible for different effects on cancer death ${ }^{33,35}$ related or not to a hormonal receptor switch..$^{20,44}$

Other limitations include the lack of information regarding radiation, hormonal therapy, the sequential chemotherapeutic regimens that were used over time, inability to use the TNM classification of breast cancer stage due to missing data on the number of affected lymph nodes at the time of diagnosis, and the retrospective nature of the study. Moreover, we did not take into account the socioeconomic gradient of this cohort. Individuals who are prescribed a statin usually represent a population of higher socioeconomic status with better access to health care, eg, screening tests, higher educational levels, and more health-conscious lifestyle, and hence more adherent to prescribed regimens, thus favoring better outcomes. However, data shown in Table 1 reveal that there were no statistically significant differences between statin users vs nonusers with respect to the stage that breast cancer was initially discovered. Slightly more patients with statin use were at clinical stage III, further enhancing our findings.

There was also limited information regarding patient body mass index and comorbidities. This could complicate our 
results, both in terms of comedication that could carry antineoplastic effects (eg, aspirin) and cancer-specific outcomes. However, if comorbidities influence cancer-specific outcomes, this was an advantage for nonusers in our study, and it does contradict our findings. Nonetheless, in this study we only evaluated cancer-specific outcomes, such as DFS. Patients who were on statin therapy had more comorbidities, which is reflected in the shorter median follow-up time (more censored data), and for this reason we did not evaluate the effects of statins in overall survival, but only estimated DFS using both log-rank comparisons and Cox's HR models.

\section{Perspectives and conclusion}

In conclusion, our small study showed that the concurrent administration of a statin in the adjuvant setting is possibly related to a lower risk of recurrence in patients with early stage breast cancer. This link was more evident in younger patients and those with tumors expressing hormone receptors. This is particularly important, given the increased life expectancy of these patients. However, the overall number of statins users was small, which makes subgroup analysis quite unreliable. Therefore, someone should be extremely cautious when interpreting the results. Further painstaking research lies ahead in order to address a number of issues. For example, at present no study of association between statin use and breast cancer recurrence has stratified analyses by tumor expression of HMGCR. It would be interesting to assess whether and to what extent receptor expression per se is associated with better outcomes in the presence of ER-positive breast cancer. ${ }^{7,46}$ Moreover, the intriguing finding of selective benefit among lipophilic statin users warrants further investigation. Genetic variation design experiments upon cellular membrane uptake of statins could be of use. In any case, following promising results from observational data, the issue of adjuvant breast cancer therapy with statins now relies upon meticulous design and results from randomized clinical trials. This is quite a challenging issue, because the duration of follow-up while patients are receiving a statin must at least be as long as the latency period of breast cancer. To date, there are no sufficient data to recommend statin administration routinely for the prevention of breast cancer relapse. However, further clinical studies in the future might be practice-changing.

\section{Disclosure}

An abstract of this manuscript was presented at the 2016 ASCO Annual Meeting, Chicago, IL, USA, and was published in the Journal of Clinical Oncology (2016;34:e13044). The authors report no conflicts of interest in this work.

\section{References}

1. Lakha F, Theodoratou E, Farrington SM, et al. Statin use and association with colorectal cancer survival and risk: case control study with prescription data linkage. BMC Cancer. 2012;12:487.

2. Garcia-Ruiz C, Morales A, Fernandez-Checa JC. Statins and protein prenylation in cancer cell biology and therapy. Anticancer Agents Med Chem. 2012;12:303-315.

3. Freed-Pastor WA, Mizuno H, Zhao X, et al. Mutant p53 disrupts mammary tissue architecture via the mevalonate pathway. Cell. 2012;148:244-258.

4. Soma MR, Corsini A, Paoletti R. Cholesterol and mevalonic acid modulation in cell metabolism and multiplication. Toxicol Lett. 1992;64-65:1-15.

5. Thurnher M, Gruenbacher G, Nussbaumer O. Regulation of mevalonate metabolism in cancer and immune cells. Biochim Biophys Acta. 2013;1831:1009-1015.

6. Clendening JW, Pandyra A, Boutros PC, et al. Dysregulation of the mevalonate pathway promotes transformation. Proc Natl Acad Sci US A. 2010;107:15051-15056.

7. Borgquist S, Jögi A, Pontén F, Rydén L, Brennan DJ, Jirström K. Prognostic impact of tumour-specific HMG-CoA reductase expression in primary breast cancer. Breast Cancer Res. 2008;10:R79.

8. Larsson O, Carlberg M. Requirement for isoprenoid-dependent posttranslational modifications in the cell-cycle progression of human breast-cancer cells. Int J Oncol. 1992;1:489-493.

9. Hilvo M, Denkert C, Lehtinen L, et al. Novel theranostic opportunities offered by characterization of altered membrane lipid metabolism in breast cancer progression. Cancer Res. 2011;71:3236-3245.

10. Cuello FM, Kato CS, Díaz SD, Owen G. Efectos de las estatinas en cáncer: ¿potencial rol en terapéutica y prevención? [Effects of statins in cancer]. Rev Med Chil. 2013;141:227-236. Spanish.

11. Singh S, Singh PP. Statin a day keeps cancer at bay. World J Clin Oncol. 2013;4:43-46.

12. Zanfardino M, Spampanato C, De Cicco R, et al. Simvastatin reduces melanoma progression in a murine model. Int J Oncol. 2013;43:1763-1770.

13. Wachtershauser A, Akoglu B, Stein J. HMG-CoA reductase inhibitor mevastatin enhances the growth inhibitory effect of butyrate in the colorectal carcinoma cell line Caco-2. Carcinogenesis. 2001;22:1061-1067.

14. Spampanato C, De Maria S, Sarnataro M, et al. Simvastatin inhibits cancer cell growth by inducing apoptosis correlated to activation of Bax and down-regulation of BCL-2 gene expression. Int J Oncol. 2012;40:935-941.

15. Denoyelle C, Vasse M, Körner M, et al. Cerivastatin, an inhibitor of HMG-CoA reductase, inhibits the signaling pathways involved in the invasiveness and metastatic properties of highly invasive breast cancer cell lines: an in vitro study. Carcinogenesis. 2001;22:1139-1148.

16. Mandal CC, Ghosh-Choudhury N, Yoneda T, Choudhury GG. Simvastatin prevents skeletal metastasis of breast cancer by an antagonistic interplay between p53 and CD44. J Biol Chem . 2011;286:11314-11327.

17. Bonovas S. Statins: do they have a potential role in cancer prevention and modifying cancer-related outcomes? Drugs. 2014;74:1841-1848.

18. Bonovas S, Lytras T, Sitaras NM. Statin use and breast cancer: do we need more evidence and what should this be? Expert Opin Drug Saf. 2014;13:271-275.

19. Pisanti S, Picardi P, Ciaglia E, D’Alessandro A, Bifulco M. Novel prospects of statins as therapeutic agents in cancer. Pharmacol Res. 2014;88:84-98.

20. Desai P, Chlebowski R, Cauley JA, et al. Prospective analysis of association between statin use and breast cancer risk in the Women's Health Initiative. Cancer Epidemiol Biomarkers Prev. 2013;22:1868-1876.

21. Cauley JA, Zmuda JM, Lui LY, et al. Lipid-lowering drug use and breast cancer in older women: a prospective study. JWomens Health (Larchmt). 2003;12:749-756.

22. Kumar AS, Esserman LJ. Statins: health-promoting agents show promise for breast cancer prevention. Clin Breast Cancer. 2005;6:455-459.

23. Demierre MF, Higgins PD, Gruber SB, Hawk E, Lippman SM. Statins and cancer prevention. Nat Rev Cancer. 2005;5:930-942. 
24. Zhong S, Zhang X, Chen L, Ma T, Tang J, Zhao J. Statin use and mortality in cancer patients: systematic review and meta-analysis of observational studies. Cancer Treat Rev. 2015;41:554-567.

25. Nielsen SF, Nordestgaard BG, Bojesen SE. Statin use and reduced cancer-related mortality. N Engl J Med. 2012;367:1792-1802.

26. Ahern TP, Pedersen L, Tarp M, et al. Statin prescriptions and breast cancer recurrence risk: a Danish nationwide prospective cohort study. J Natl Cancer Inst. 2011;103:1461-1468.

27. Boudreau DM, Yu O, Chubak J, et al. Comparative safety of cardiovascular medication use and breast cancer outcomes among women with early stage breast cancer. Breast Cancer Res Treat. 2014;144:405-416.

28. Chae YK, Valsecchi ME, Kim J, et al. Reduced risk of breast cancer recurrence in patients using ACE inhibitors, ARBs, and/or statins. Cancer Invest. 2011;29:585-593.

29. Kwan ML, Habel LA, Flick ED, Quesenberry CP, Caan B. Post-diagnosis statin use and breast cancer recurrence in a prospective cohort study of early stage breast cancer survivors. Breast Cancer Res Treat. 2008;109:573-579.

30. Nickels S, Vrieling A, Seibold P, et al. Mortality and recurrence risk in relation to the use of lipid-lowering drugs in a prospective breast cancer patient cohort. PLoS One. 2013;8:e75088.

31. Park JH, McMillan DC, Horgan PG, Roxburgh CS. The impact of antiinflammatory agents on the outcome of patients with colorectal cancer. Cancer Treat Rev. 2014;40:68-77.

32. Bonovas S, Filioussi K, Tsavaris N, Sitaras NM. Use of statins and breast cancer: a meta-analysis of seven randomized clinical trials and nine observational studies. J Clin Oncol. 2005;23:8606-8612.

33. Brewer TM, Masuda H, Liu DD, et al. Statin use in primary inflammatory breast cancer: a cohort study. Br J Cancer. 2013;109:318-324.

34. Cardwell CR, Hicks BM, Hughes C, Murray LJ. Statin use after diagnosis of breast cancer and survival: a population-based cohort study. Epidemiology. 2015;26:68-78.

35. Desai P, Lehman A, Chlebowski RT, et al. Statins and breast cancer stage and mortality in the Women's Health Initiative. Cancer Causes Control. 2015;26:529-539.

36. Murtola TJ, Visvanathan K, Artama M, Vainio H, Pukkala E. Statin use and breast cancer survival: a nationwide cohort study from Finland. PLoS One. 2014;9:e110231.

37. Graaf MR, Richel DJ, van Noorden CJ, Guchelaar HJ. Effects of statins and farnesyltransferase inhibitors on the development and progression of cancer. Cancer Treat Rev. 2004;30:609-641.
38. Blackwell KL, Haroon ZA, Shan S, et al. Tamoxifen inhibits angiogenesis in estrogen receptor-negative animal models. Clin Cancer Res. 2000;6:4359-4364.

39. Dulak J, Jozkowicz A. Anti-angiogenic and anti-inflammatory effects of statins: relevance to anti-cancer therapy. Curr Cancer Drug Targets. 2005;5:579-594.

40. Mor G, Sapi E, Abrahams VM, et al. Interaction of the estrogen receptors with the Fas ligand promoter in human monocytes. J Immunol. 2003;170:114-122.

41. Sleijfer S, van der Gaast A, Planting AS, Stoter G, Verweij J. The potential of statins as part of anti-cancer treatment. Eur J Cancer. 2005;41:516-522.

42. Nelson ER, Wardell SE, Jasper JS, et al. 27-Hydroxycholesterol links hypercholesterolemia and breast cancer pathophysiology. Science. 2013;342:1094-1098

43. Zhou Q, Atadja P, Davidson NE. Histone deacetylase inhibitor LBH589 reactivates silenced estrogen receptor alpha (ER) gene expression without loss of DNA hypermethylation. Cancer Biol Ther. 2007;6:64-69.

44. Kumar AS, Benz CC, Shim V, Minami CA, Moore DH, Esserman LJ. Estrogen receptor-negative breast cancer is less likely to arise among lipophilic statin users. Cancer Epidemiol Biomarkers Prev. 2008; 17:1028-1033.

45. Byers T. Statins, breast cancer, and an invisible switch? Cancer Epidemiol Biomarkers Prev. 2008;17:1026-1027.

46. Brennan DJ, Laursen H, O'Connor DP, et al. Tumor-specific HMG-CoA reductase expression in primary premenopausal breast cancer predicts response to tamoxifen. Breast Cancer Res. 2011;13:R12.

47. Anders CK, Hsu DS, Broadwater G, et al. Young age at diagnosis correlates with worse prognosis and defines a subset of breast cancers with shared patterns of gene expression. J Clin Oncol. 2008;26:3324-3330.

48. Lund MJ, Trivers KF, Porter PL, et al. Race and triple negative threats to breast cancer survival: a population-based study in Atlanta, GA. Breast Cancer Res Treat. 2009;113:357-370.

49. Shannon C, Smith IE. Breast cancer in adolescents and young women. Eur J Cancer. 2003;39:2632-2642.

50. Garwood ER, Kumar AS, Baehner FL, et al. Fluvastatin reduces proliferation and increases apoptosis in women with high grade breast cancer. Breast Cancer Res Treat. 2010;119:137-144.

51. Bjarnadottir O, Romero Q, Bendahl PO, et al. Targeting HMG-CoA reductase with statins in a window-of-opportunity breast cancer trial. Breast Cancer Res Treat. 2013;138:499-508.
Breast Cancer - Targets and Therapy

\section{Publish your work in this journal}

Breast Cancer - Targets and Therapy is an international, peerreviewed open access journal focusing on breast cancer research, identification of therapeutic targets and the optimal use of preventative and integrated treatment interventions to achieve improved outcomes, enhanced survival and quality of life for the cancer patient.

\section{Dovepress}

The manuscript management system is completely online and includes a very quick and fair peer-review system, which is all easy to use. Visit http://www.dovepress.com/testimonials.php to read real quotes from published authors. 